RESEARCH ARTICLE

\title{
Insulin Regulates Circulating Omentin, Fatty Acid Binding Protein-4 and Chemerin, but not Irisin in Man
}

\author{
Nandita K Shankar ${ }^{1}$, Clayton A Dehn ${ }^{2 *}$, Robert V Considine ${ }^{3}$ and Helmut O Steinberg ${ }^{4}$ \\ ${ }^{1}$ Tulane University, USA \\ ${ }^{2}$ High Point Clinical Trials Center, USA \\ ${ }^{3}$ Division of Endocrinology, Department of Medicine, Indiana University School of Medicine, Indianapolis, USA \\ ${ }^{4}$ Division of Endocrinology, Department of Medicine, University of Tennessee Health Science Center, USA
}

*Corresponding author: Clayton A Dehn, High Point Clinical Trials Center, 4160 Mendenhall Oaks Pkwy, Suite 105, High Point, NC 27265, USA, Tel: 336-841-0700-ext 2522,E-mail: cdehn@highpointctc.com

\begin{abstract}
Omentin, fatty acid binding protein-4 (FABP-4), chemerin and irisin are chemokines whose circulating levels are altered in insulin resistant states. However, there is limited insight into function of these chemokines as well as the potential role of insulin in the modulation of these chemokines. We tested the hypothesis that insulin alters these chemokines by measuring changes in their circulating levels in response to elevated insulin in a group of healthy non-obese subjects. Nine (4 males and 5 females) healthy non-obese (BMI $\left.22.0 \pm 1.8 \mathrm{~kg} / \mathrm{m}^{2}\right)$ volunteers (27 \pm 4 years of age) were enrolled in an IRB-approved clinical trial. Following an overnight fast, all subjects underwent a hyperinsulinemic euglycemic clamp procedure at two physiologically relevant doses of insulin administered in sequence. Subjects received $10 \mathrm{mU} / \mathrm{m}^{2} / \mathrm{min}$ of insulin for 180 minutes during the low dose period (LD), followed by the high dose period (HD) at $40 \mathrm{mU} / \mathrm{m}^{2} / \mathrm{min}$ of insulin for an additional 180 minutes. Subjects underwent serial sampling for insulin, omentin, FABP-4, chemerin and irisin at baseline and steady-state of each period of the clamp. Significant $(p<0.01)$ decreases were observed in omentin and FABP-4 in response to hyperinsulinemia. Chemerin and irisin were measured in 6 subjects. Significant $(p<0.01)$ decreases were observed in chemerin in response to hyperinsulinemia. In contrast, irisin did not change in response to exogenous insulin. Our results show that hyperinsulinemia suppresses omentin, FABP-4 and chemerin, but not irisin in healthy non-obese humans, suggesting a potential role for insulin in regulating omentin, FABP-4 and chemerin in man.
\end{abstract}

\section{Introduction}

Omentin, fatty acid binding protein-4 (FABP-4), chemerin and irisin are adipocyte and myocyte-derived chemokines [1-5]. These chemokines are reported to be altered in insulin resistance and have even been proposed to mediate of some of the features of insulin resistance [2-5]. However, little is known about the regulation and function of these chemokines. Endogenous hyperinsulinemia is a hallmark of the insulin resistant state. Therefore, it is reasonable to invoke a potential role for this compensatory hyperinsulinemia in the regulation of these metabolically relevant chemokines. In particular, understanding the effect of elevated insulin levels in the range observed in insulin resistance in such regulation could be especially informative. We hypothesized that insulin does have a role in altering these chemokines. We tested our hypothesis by measuring changes in circulating levels of these chemokines in a group of non-obese healthy volunteers in response to elevated insulin levels in the setting of a hyperinsulinemic euglycemic clamp procedure. Our results show that hyperinsulinemia suppresses omentin, FABP-4 and chemerin, but not irisin in healthy humans, suggesting a potential role for insulin in regulating omentin, FABP-4 and chemerin.

\section{Methods}

The current study was conducted under a protocol that was approved by an Institutional Review Board. After providing written informed consent, and following successful screening, 9 non-obese healthy volunteers were enrolled in the trial. Following an overni-

Citation: Shankar NK, Dehn CA, Considine RV, Steinberg HO (2018) Insulin Regulates Circulating Omentin, Fatty Acid Binding Protein-4 and Chemerin, but not Irisin in Man. Int J Diabetes Clin Res 5:090. doi. org/10.23937/2377-3634/1410090

Accepted: July 21, 2018: Published: July 23, 2018

Copyright: (C) 2018 Shankar NK, et al. This is an open-access article distributed under the terms of the Creative Commons Attribution License, which permits unrestricted use, distribution, and reproduction in any medium, provided the original author and source are credited. 
ght fast, all subjects underwent a 360 -minute two-step hyperinsulinemic euglycemic clamp procedure with two doses of insulin (Insulin lispro) administered in sequence. Subjects received $10 \mathrm{mU} / \mathrm{m}^{2} / \mathrm{min}$ of insulin from $\mathrm{T}=$ 0 to 180 minutes during the low dose period, followed by an insulin infusion at $40 \mathrm{mU} / \mathrm{m}^{2} / \mathrm{min}$ of insulin from $\mathrm{T}=180$ to 360 minutes during the high dose period. Plasma glucose was measured every 5 minutes throughout the clamp using a YSI glucose analyzer at the bedside. Exogenous glucose was administered as a continuous variable intravenous infusion of $20 \%$ dextrose in water (D20\%W), and glucose infusion rates adjusted to maintain an approximate target plasma glucose of $90 \mathrm{mg} / \mathrm{dL}$ throughout the clamp procedure. Subjects underwent serial sampling for insulin at baseline and during steady-state of the low and high dose periods of the clamp procedure. Insulin was measured using a RIA (Millipore). The average of four insulin measurements obtained 20

Table 1: Baseline Characteristics.

\begin{tabular}{|l|l|}
\hline Parameter & Mean \pm SD \\
\hline Age & $27 \pm 4$ \\
\hline BMI $\left(\mathrm{kg} / \mathrm{m}^{2}\right)$ & $22 \pm 1.8$ \\
\hline Fasting Glucose $(\mathrm{mg} / \mathrm{dL})$ & $86.5 \pm 4.4$ \\
\hline Fasting Insulin $(\mu \mathrm{lU} / \mathrm{mL})$ & $6.9 \pm 2.3$ \\
\hline Total Cholesterol $(\mathrm{mg} / \mathrm{dL})$ & $161.1 \pm 2.5$ \\
\hline Systolic BP $(\mathrm{mmHg})$ & $112 \pm 11$ \\
\hline Diastolic BP $(\mathrm{mmHg})$ & $60 \pm 7$ \\
\hline
\end{tabular}

minutes apart during steady state conditions was used for analysis. Plasma omentin and FABP-4 levels were measured in 9 subjects at baseline and during both low and high dose periods of the clamp procedure. Chemerin and irisin levels were measured in 6 subjects at baseline and during the low and high periods of the clamp procedure. All the chemokines were measured using commercial ELISA kits (Biovendor). Circulating omentin, FABP-4, chemerin and irisin at high and low dose steady state of the clamp procedure were compared to that at baseline in a step-wise procedure using paired-t tests. Data from the clamp procedure are expressed as mean \pm SEM.

\section{Results}

Baseline characteristics (Table 1 ) of the subjects ( $N$ = 9; 4 males and 5 females) were as follows, with data expressed as mean \pm SD: age: $27 \pm 4$ years, BMI of 22.0 $\pm 1.8 \mathrm{~kg} / \mathrm{m}^{2}$, fasting glucose: $86.5 \pm 4.4 \mathrm{mg} / \mathrm{dL}$, fasting insulin: $6.9 \pm 2.3 \mu \mathrm{U} / \mathrm{mL}$, total cholesterol levels: 161.1 $\pm 25.2 \mathrm{mg} / \mathrm{dL}$, systolic blood pressure: $112 \pm 11 \mathrm{mmHg}$ and diastolic blood pressure: $60 \pm 7 \mathrm{mmHg}$.

\section{Omentin and FABP-4}

During the low dose portion of the clamp, at steady-state, plasma glucose levels were $89.7 \pm 0.7 \mathrm{mg} / \mathrm{dL}$ and plasma insulin levels were $22.4 \pm 1.0 \mu \mathrm{U} / \mathrm{mL}$, while glucose

Table 2: Insulin Infusion (steady state).

\begin{tabular}{|l|l|l|l|l|l|}
\hline Parameter & Baseline & Low Dose & & \multicolumn{2}{l|}{ High Dose } \\
\cline { 2 - 6 } & Mean \pm SD & Mean \pm SD & $\Delta$ From Baseline & Mean \pm SD & $\Delta$ From Baseline \\
\hline Omentin $(\mathrm{ng} / \mathrm{mL})$ & $484 \pm 41$ & $418 \pm 42$ & $\mathrm{p}<0.01$ & $372 \pm 36$ & $\mathrm{p}<0.01$ \\
\hline FAB-P4 $(\mathrm{ng} / \mathrm{mL})$ & $9.5 \pm 1.2$ & $7.5 \pm 1.2$ & $\mathrm{p}<0.01$ & $7.7 \pm 1.2$ & $\mathrm{p}<0.01$ \\
\hline Chemerin $(\mathrm{ng} / \mathrm{mL})$ & $162 \pm 36.6$ & $143 \pm 32.1$ & $\mathrm{p}<0.01$ & $138.9+25.9$ & $\mathrm{p}<0.01$ \\
\hline Irisin $(\mu \mathrm{g} / \mathrm{mL})$ & $4.7 \pm 0.9$ & $4.7 \pm 0.7$ & $\mathrm{p}=\mathrm{NS}$ & $4.7+0.6$ & $\mathrm{p}=\mathrm{NS}$ \\
\hline
\end{tabular}

\section{Ometin Suppression in Response to Insulin}

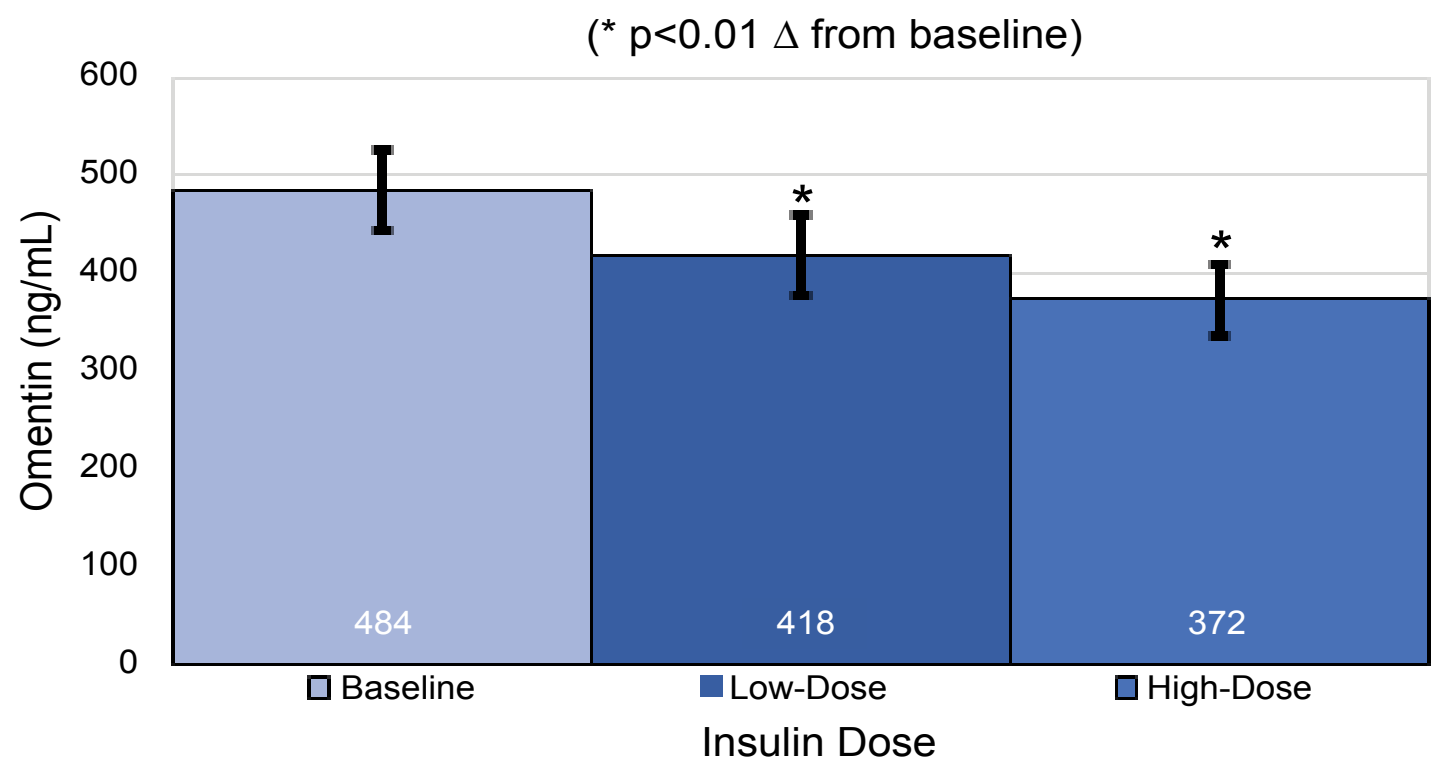

Figure 1: Absolute values and standard deviations for plasma omentin levels in $\mathrm{ng} / \mathrm{mL}$ at baseline, under low dose hyperinsulinemia and under high dose hyperinsulinemia. ${ }^{*} p<0.01$ over baseline for mean and SEM. 
infusion rates were $3.5 \pm 0.4 \mathrm{mg} / \mathrm{kg} / \mathrm{min}$ (Table 2, Figure 1 and Figure 2). During the high dose portion of the clamp, at steady-state, plasma glucose levels were $92.6 \pm 1.3 \mathrm{mg} /$ $\mathrm{dL}$ and plasma insulin levels were $83.4 \pm 1.6 \mu \mathrm{U} / \mathrm{mL}$, while glucose infusion rates were $11.5 \pm 0.8 \mathrm{mg} / \mathrm{kg} / \mathrm{min}$.

At baseline, prior to initiation of the clamp procedu$r e$, and in the fasted state, omentin levels were $484 \pm 41$ $\mathrm{ng} / \mathrm{mL}$. A significant $(\mathrm{p}<0.01)$ decrease from baseline in plasma omentin levels was observed at steady-state of the low dose portion of the clamp, reaching $418 \pm 42$ $\mathrm{ng} / \mathrm{mL}$, with a further decrease at steady-state of the high dose portion of the clamp to $372 \pm 36 \mathrm{ng} / \mathrm{mL}$ ( $p<$ 0.01 over baseline).

At baseline, prior to initiation of the clamp procedu$r e$, and in the fasted state, FABP-4 levels were $9.5 \pm 1.2$ $\mathrm{ng} / \mathrm{mL}$. A significant $(\mathrm{p}<0.01)$ decrease from baseline in plasma FABP-4 levels was observed at steady-state of the low dose portion of the clamp, reaching $7.5 \pm 1.2$ $\mathrm{ng} / \mathrm{mL}$, with no further lowering at steady-state of the high dose portion of the clamp $(7.7 \pm 1.2 \mathrm{ng} / \mathrm{mL} ; \mathrm{p}<0.01$ over baseline).

\section{Chemerin and Irisin}

During the low dose portion of the clamp, at steady-state, plasma glucose levels were $90.0 \pm 1.7 \mathrm{mg} / \mathrm{dL}$ and plasma insulin levels were $22.1 \pm 1.8 \mu \mathrm{U} / \mathrm{mL}$, while glucose infusion rates were $2.3 \pm 0.2 \mathrm{mg} / \mathrm{kg} / \mathrm{min}$ (Table 2). During the high dose portion of the clamp, at steady-state, plasma glucose levels were $93.7 \pm 3.0 \mathrm{mg} / \mathrm{dL}$ and plasma insulin levels were $78.2 \pm 1.8 \mu \mathrm{U} / \mathrm{mL}$, while glucose infusion rates were $9.7 \pm 0.8 \mathrm{mg} / \mathrm{kg} / \mathrm{min}$.

At baseline in the fasted state, prior to initiation of the clamp procedure, chemerin levels were $162 \pm 36.6$ $\mathrm{ng} / \mathrm{mL}$. A significant $(\mathrm{p}<0.01)$ decrease from baseline in plasma chemerin levels was observed at steady-state of the low dose portion of the clamp, reaching $143 \pm 32.1$ $\mathrm{ng} / \mathrm{mL}$, with no further significant decrease during the high dose portion of the clamp.

At baseline, in the fasted state prior to initiation of the clamp procedure, irisin levels were $4.7 \pm 0.9 \mu \mathrm{g} / \mathrm{mL}$, and were $4.7 \pm 0.7 \mu \mathrm{g} / \mathrm{mL}$ under low dose and $4.7 \pm 0.6$ $\mu \mathrm{g} / \mathrm{mL}$ under high dose hyperinsulinemia.

\section{Discussion}

We evaluated the effects of experimental hyperinsulinemia recapitulating the range from fasted to fed states, on a background of euglycemia, on select chemokines in a group of healthy non-obese subjects. Our findings indicate a possible role for insulin in the regulation of several of the adipokines studied.

Omentin levels in circulation and in visceral (omental) adipose tissue, as well as omentin mRNA concentrations have been reported to be decreased in obesity, type 2 diabetes and polycystic ovary syndrome [1-3]. Furthermore, in one study plasma omentin levels correlated inversely with BMI, fasting insulin, HOMA-IR and other features of the metabolic syndrome [5]. Our findings show that insulin suppressed omentin levels by 13 to $23 \%$ across the range of clinically relevant levels of insulin and does so in the presence of fixed glycemia. While the relationship with omentin and insulin was numerically dose-dependent, it did not reach statistical significance. Tan, et al. showed in a group of 6 subjects that exogenous insulin and glucose administered together at fixed rates suppress plasma omentin levels [6]. Our data are concordant with Tan, et al. in the directionality of effect observed, and its relatively rapid onset and peak. In addition, since our study was performed in the con-

\section{FABP-4 Suppression in Response to Insulin}

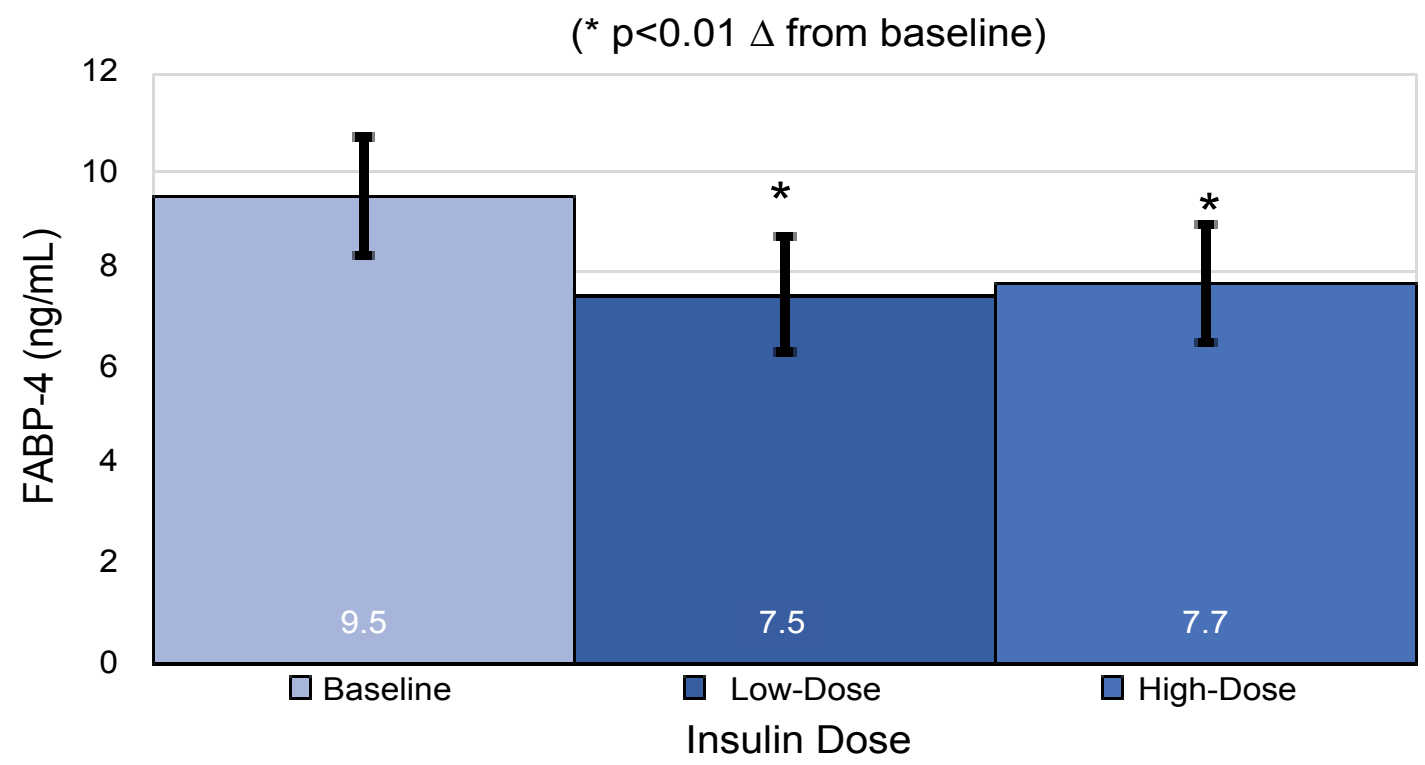

Figure 2: Absolute values and standard deviations for plasma FABP-4 levels in $\mathrm{ng} / \mathrm{mL}$ at baseline, under low dose hyperinsulinemia and under high dose hyperinsulinemia. ${ }^{*} p<0.01$ over baseline for mean and SEM. 
text of clamped glucose levels, we were able to show that the suppressive effect of insulin on omentin occurs even when glucose is kept stable. Our findings therefore clearly demonstrate that insulin is a potential driver for omentin regulation under conditions where glucose is stable.

Increases in basal FABP-4 levels have been reported in obesity and insulin resistant states [4]. We found that clinically relevant hyperinsulinemia significantly suppressed levels of FABP-4 by approximately $18 \%$. We did not observe a dose-dependence in the suppression of FABP-4 in response to insulin suggesting that the effect appears to be maximal even at the exposures achieved during the low dose insulin infusion period. $\mathrm{Wu}$, et al. reported a decrease in FABP-4 levels in response to 2 hours of hyperinsulinemia in a group of overweight humans [7]. Our findings confirm these results and extend them to non-obese insulin sensitive subjects as well. FABP-4 has been proposed to play a role in facilitating fatty acid and glucose-dependent insulin secretion [4,7]. It is possible that the ability of insulin to lower FABP-4 may be part of a feedback loop to prevent hyperinsulinemia $[4,7]$.

The current body of literature around chemerin in the context of metabolic disease suggests a variety of potential biological functions for the adipokine [8]. There are studies showing association of elevated basal chemerin levels in obesity, insulin resistance, the metabolic syndrome and even diabetes $[9,10]$. There are also reports indicating that chemerin may have anti-inflammatory properties, potentially enable adipocyte differentiation and angiogenesis, and finally, a role in enhancing insulin secretion [11]. Our results indicate that insulin across the range of routinely encountered levels, suppresses chemerin levels by approximately $12 \%$ in a non-obese healthy subject population. Similar to FABP4, we did not observe a dose-dependence in the suppression of chemerin in response to insulin. Again, this may suggest that the effect appears to be maximal even at the exposures achieved during the low dose insulin infusion period. In contrast to our findings, Tan, et al. report an increase in chemerin levels in the context of a co-infusion of insulin and glucose in a group of 6 healthy subjects [12]. In our experiment, we maintained glucose at a fixed euglycemic level, and our findings may reflect the effect of insulin alone to lower chemerin, in contrast to the combined effect of elevated insulin and variable plasma glucose as reported by Tan, et al. [12]. The same study [12] also reported that metformin treatment in a group of subjects with polycystic ovary syndrome leads to a reduction in chemerin levels, accompanied by an improvement in insulin resistance and a lowering of glucose levels. It is important to recognize that a number of hormones changed in response to metformin, each of which may have contributed to the observed effect. Esteghamati, et al. reported decreases in chemerin levels in response to both pioglitazone and metformin along with decreases in glucose levels and improvement in insulin resistance in patients with poorly controlled Type 2 diabetes [13]. Factors other than improvement of insulin resistance (e.g. improvement in glycemia) may have contributed to the observed effect. Therefore, our observation of a decrease in chemerin in a healthy population in response to isolated hyperinsulinemia with tightly controlled glucose is a novel finding in that it describes the effect of insulin alone on chemerin. Additional human in vivo studies are required to better understand the role and regulation of chemerin in metabolic conditions.

Levels of irisin in circulation, skeletal muscle and adipose tissue are reported to be decreased in insulin resistance and Type 2 diabetes [14-17]. There are also reports of changes in irisin levels in response to interventions such as exercise [18]. However, there are no reports of the effect of insulin on irisin. We show for the first time that insulin does not appear to play a role in regulation of circulating irisin. It is possible that the small sample size in which we measured irisin was unable to detect a change in response to insulin. However, since we were able to detect changes in other chemokines in similar sample sizes of the same population, our data likely represent a true finding. Nevertheless, confirmatory data from additional studies, particularly by other investigators would add considerable value.

Taken together, our results demonstrate that in a group of healthy non-obese subjects, insulin has a regulatory role on circulating omentin, FABP-4 and chemerin, but not on irisin levels. Notably, we have been able to show that the effect of insulin to regulate these adipokines is robust and independent of changes in prevailing glycemia. The experimental hyperinsulinemia in the current study recapitulates the endogenous compensatory hyperinsulinemia that is a hallmark of insulin resistance. Therefore, our findings of insulin's effect to suppress circulating omentin, FABP-4 and chemerin suggest that the compensatory hyperinsulinemia of insulin resistance may in part lead to the lower levels of these adipokines in various insulin resistant states. Future work to confirm the translation of these effects into obese and dysmetabolic populations, such as those with Type 2 diabetes would considerably enhance our understanding of these adipokines.

\section{Statement of Author's Contributions}

- NKS performed analysis of samples, interpreted data, and wrote the manuscript.

- CAD supervised the project development, performed the clinical conduct, analyzed samples.

- Interpreted data and critically reviewed and edited the manuscript.

- RVC Analyzed samples, interpreted data critically reviewed and edited the manuscript. 
- HOS contributed to the project development, provided medical oversight, analysis of samples, interpreted data, and critically reviewed and edited the manuscript.

This work was not supported with funding from the National Institutes of Health (NIH), Wellcome Trust, or other disclosable organization.

\section{References}

1. Garrett M Brodeur, Radhika lyer, Jamie L Croucher, Tiangang Zhuang, Mayumi Higashi, et al. (2014) Therapeutic targets for neuroblastomas. Expert Opin Ther Targets 18: 277-292.

2. Rabe K, Lehrke M, Parhofer KG, Broedl UC (2008) Adipokines and Insulin Resistance. Mol Med 14: 741-751.

3. Rondinone CM (2006) Adipocyte-derived hormones, cytokines, and mediators. Endocr 29: 81-90.

4. Nakamura R, Okura T, Fujioka Y, Sumi K, Matsuzawa K, et al. (2017) Serum fatty acid-binding protein 4 (FABP4) concentration is associated with insulin resistance in peripheral tissues, A clinical study. PLoS One 12: e0179737.

5. de Souza Batista CM, Yang RZ, Lee MJ, Glynn NM, Yu DZ, et al. (2007) Omentin Plasma Levels and Gene Expression Are Decreased in Obesity. Diabetes 56: 1655-1661.

6. Tan BK, Adya R, Farhatullah S, Lewandowski KC, Hare $\mathrm{PO}$, et al. (2008) Omentin-1, a novel adipokine, is decreased in overweight insulin-resistant women with polycystic ovary syndrome: Ex vivo and in vivo regulation of omentin-1 by insulin and glucose. Diabetes 57: 801-808.

7. Wu LE, Samocha-Bonet D, Whitworth PT, Fazakerley DJ, Turner N, et al. (2014) Identification of fatty acid binding protein 4 as an adipokine that regulates insulin secretion during obesity. Molecular Metabolism 3: 465-473.

8. Rourke JL, Dranse HJ, Sinal CJ (2013) Towards an integrative approach to understanding the role of chemerin in human health and disease. Obes Rev 14: 245-262.
9. Bozaoglu K, Bolton K, McMillan J, Zimmet P, Jowett J, et al. (2007) Chemerin Is a Novel Adipokine Associated with Obesity and Metabolic Syndrome. Endocrinology 148: 4687-4694.

10. Sledzinski T, Korczynska J, Hallmann A, Kaska L, Proczko-Markuszewska M, et al. (2013) The increase of serum chemerin concentration is mainly associated with the increase of body mass index in obese, non-diabetic subjects. J Endocrinol Invest 36: 428-434.

11. Ernst MC, Sinal CJ (2010) Chemerin: At the crossroads of inflammation and obesity. Trends in Endocrinology and Metabolism 21: 660-667.

12. Tan BK, Chen J, Farhatullah S, Adya R, Kaur J, et al. (2009) Insulin and metformin regulate circulating and adipose tissue chemerin. Diabetes 58: 1971-1977.

13. Esteghamati A, Ghasemiesfe M, Mousavizadeh M, Noshad S, Nakhjavani (2014) M Pioglitazone and metformin are equally effective in reduction of chemerin in patients with type 2 diabetes. J Diabetes Investig 5: 327-332.

14. Perakakis N, Triantafyllou GA, Fernández-Real JM, Huh JY, Park KH, et al. (2017) Physiology and role of irisin in glucose homeostasis. Nature Reviews Endocrinology 13: 324-337.

15. Novelle MG, Contreras C, Romero-Picó A, López M, Diéguez C (2013) Irisin, Two Years Later. International Journal of Endocrinology 2013: 8.

16. Park KH, Zaichenko L, Brinkoetter M, Thakkar B, Sahin-Efe A, et al. (2013) Circulating irisin in relation to insulin resistance and the metabolic syndrome. J Clin Endocrinol Metab 98: 4899-4907.

17. Moreno-Navarrete JM, Ortega F, Serrano M, Guerra E, Pardo G (2013) Irisin is expressed and produced by human muscle and adipose tissue in association with obesity and insulin resistance. J Clin Endocrinol Metab 98: 769-778.

18. LeBlanc DRB, Rioux BV, Pelech C, Moffatt TL, Kimber DE, et al. (2017) Exercise-induced irisin release as a determinant of the metabolic response to exercise training in obese youth: The EXIT trial. Physiol Rep 5: 13539. 